\title{
Drosophila's outdoor schedule
}

Fly rhythms under natural light and temperature differ from those in the lab.

A fruit fly in a lab does not sense the sunrise. There are no lengthening days, cool mornings or hot afternoons. Under typical laboratory conditions, lights are flicked on or off every 12 hours; temperature is a constant $25^{\circ} \mathrm{C}$. These are not the most natural conditions for studying the 24-hour cycles of behavior known as circadian rhythms, but they are what researchers have used for decades.

In the lab, flies have a sharp burst of activity that starts just before the lights turn on in the morning; a second burst in activity comes in the evening, just before the lights go off. Aberrations in these patterns were crucial to discovering several 'clock genes' that govern rhythms for sleep-wake-activity patterns.

In light of these long-established observations, flies are thought to be adapted for activity bursts around dawn and dusk. There is even a set of neuronal clusters that determines morning activity and another that controls evening activity. But new research shows that flies living outdoors keep a different schedule.

From spring to autumn, research teams led by Rodolfo Costa at the University of Padova in Italy and Charalambos Kyriacou at the University of Leicester in the UK kept flies outdoors, where they were exposed to daily light and temperature fluctuations at $46^{\circ}$ and $52^{\circ}$ latitude, respectively. In addition to bursts of activity around dusk and dawn, flies maintained al fresco have a third burst, in the afternoon. More than half of the flies perked up in the afternoon at average daily temperatures of $20^{\circ} \mathrm{C}$; nearly all did at 27 ${ }^{\circ} \mathrm{C}$. In fact, when total daily levels of activity were considered, less than a quarter of the flies' activity occurred around dawn and dusk. Although fruit flies have gen- erally been labeled 'crepuscular'(meaning most active in twilight), this finding indicates that these insects would be better classified as diurnal, says Costa.

As the weather got hotter, the flies' morning burst of activity came earlier and the evening burst grew later, correlating with the lengthening days. English summer days have 3-4 hours' more daylight than the corresponding Italian days further south, but the timing of the English and Italian flies' activity was largely dependent on average temperatures. This shows, says Costa, "the dominance of temperature over photo-period in nature, a result at odds with most laboratory studies." However, temperature is not the only factor: if flies were covered up so that they could not sense the coming dawn, their morning bursts in activity were delayed.

Some mutant flies with off-sync behavior in the lab showed regular patterns outdoors and generally behaved similarly to their wild-type counterparts. Circadian rhythms are thought to help organisms tailor activities to the most suitable times of day as well as adapt to gradual seasonal changes. Internal cues may be particularly important for regulating internal organs, whereas other activity can take its cue from external factors, says Costa. "Our study suggests that behavior, which is the brain's way of changing its environment, doesn't necessarily need to anticipate regular environmental changes; it can simply react to them."

Whereas temperature and twilight cues get flies up in the morning, internal clockderived cues seem to regulate afternoon and evening behavior. The PERIOD gene affects timing of circadian rhythms: the 'long' mutants have more time between their morning and evening activity periods, whereas the 'short' mutants have less. Outdoors, both types of mutants had their

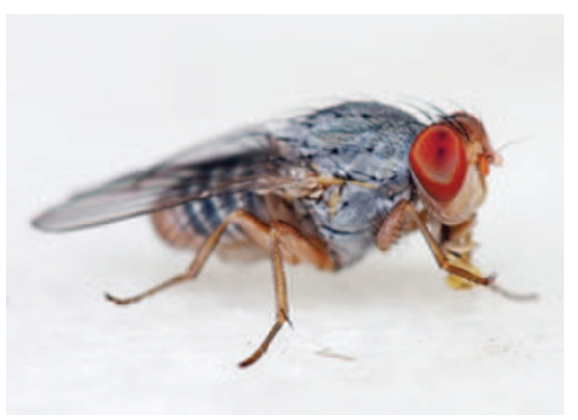

Flies' daily rhythms differ in lab and outdoors

morning burst of activity at the same time, but their afternoon and evening activities were different, with the 'short' mutants' activity bursts occurring earlier and 'long' mutants' bursts occurring later.

Costa's team was able to replicate patterns observed under outdoor conditions in the laboratory by simulating natural daylight and temperature variations, but doing so was difficult. An electronics whiz designed a panel of programmable whitelight LEDs that can vary light intensity over six or more orders of magnitude. Also, if the temperature changes were not very smooth, the flies' behavior was affected.

Experiments in animal behavior could benefit from conditions closer to animals' natural environments, says Costa. Not only will these adjustments provide better interpretation of what behaviors are adaptive, they could also improve animal welfare. "The results of our experiments could help make animals' environments healthier and more comfortable through simple measures like changing light levels and temperatures gradually."

\section{Monya Baker}

\section{RESEARCH PAPERS}

Vanin, S. et al. Unexpected features of Drosophila circadian behavioural rhythms under natural conditions. Nature 384, 371-375 (2012). 